\title{
Correction to: Integrative analysis of loss-of- function variants in clinical and genomic data reveals novel genes associated with cardiovascular traits
}

Benjamin S. Glicksberg ${ }^{1,2,14}$, Letizia Amadori ${ }^{1,3}$, Nicholas K. Akers' ${ }^{1}$, Katyayani Sukhavasi ${ }^{4}$, Oscar Franzén 1,5,15, Li Li ${ }^{1,2}$, Gillian M. Belbin ${ }^{1,6}$, Kristin L. Ayers ${ }^{1,7}$, Khader Shameer ${ }^{1,2}$, Marcus A. Badgeley ${ }^{1,2}$, Kipp W. Johnson ${ }^{1,2}$, Ben Readhead ${ }^{1,2}$, Bruce J. Darrow ${ }^{3}$, Eimear E. Kenny ${ }^{6,8}$, Christer Betsholtz $^{9}$, Raili Ermel ${ }^{10}$, Josefin Skogsberg ${ }^{11}$, Arno Ruusalepp ${ }^{5,9}$, Eric E. Schadt 1,2,5, Joel T. Dudley ${ }^{1,2,12}$, Hongxia Ren ${ }^{13}$, Jason C. Kovacic ${ }^{3}$, Chiara Giannarelli, ${ }^{1,3}$, Shuyu D. Li $1^{1,7^{*}}$, Johan L. M. Björkegren ${ }^{1,4,5,11^{*}}$ and Rong Chen ${ }^{1,7^{*}}$

\section{Correction to: BMC Med Genomics https://doi.org/10.1186/s12920-019-0542-3}

\section{Correction}

After publication of this supplement article [1], it was brought to our attention that the eighth author's name was incorrectly spelt. The correct name should be Kristin L. Ayers.

\footnotetext{
Author details

'Department of Genetics and Genomic Sciences, The Icahn Institute for Genomics and Multiscale Biology, Icahn School of Medicine at Mount Sinai, One Gustave L. Levy Place, New York, NY 10029, USA. ${ }^{2}$ The Institute for Next Generation Healthcare, Icahn School of Medicine at Mount Sinai, One Gustave L. Levy Place, New York, NY 10029, USA. ${ }^{3}$ Cardiovascular Research Center and Cardiovascular Institute, Icahn School of Medicine at Mount Sinai, One Gustave L. Levy Place, New York, NY 10029, USA. ${ }^{4}$ Department of Pathophysiology, Institute of Biomedicine and Translation Medicine, University of Tartu, Biomeedikum, Ravila 19, 50411 Tartu, Estonia. ${ }^{5} \mathrm{Clinical}$ Gene Networks AB, Jungfrugatan 10, 11444 Stockholm, Sweden. ${ }^{6}$ Charles Bronfman Institute of Personalized Medicine, Icahn School of Medicine at Mount Sinai, One Gustave L. Levy Place, New York, NY 10029, USA. 'Sema4, a Mount Sinai venture, Stamford, CT 06902, USA. ${ }^{8}$ Department of Preventive Medicine, Icahn School of Medicine at Mount Sinai, One Gustave L. Levy Place, New York, NY 10029, USA. ${ }^{9}$ Department of Immunology, Genetics and Pathology, Uppsala University, 75185 Uppsala, Sweden. ${ }^{10}$ Department of Cardiac Surgery, Tartu University Hospital, 1a Ludwig Puusepa Street, 50406 Tartu, Estonia. ${ }^{11}$ Integrated Cardio Metabolic Centre, Department of Medicine, Karolinska Institutet, Karolinska Universitetssjukhuset Huddinge, 141 86 Stockholm, Sweden. ${ }^{12}$ Department of Health Policy and Research, Icahn School of Medicine at Mount Sinai, One Gustave L. Levy Place, New York, NY 10029, USA. ${ }^{13}$ Department of Pediatrics, Herman B Wells Center for
}

\footnotetext{
*Correspondence: shuyudan.li@mssm.edu; johan.bjorkegren@mssm.edu; rong.chen@mssm.edu

'Department of Genetics and Genomic Sciences, The Icahn Institute for Genomics and Multiscale Biology, Icahn School of Medicine at Mount Sinai, One Gustave L. Levy Place, New York, NY 10029, USA

Full list of author information is available at the end of the article
}

PediatricResearch, Center for Diabetes and Metabolic Diseases, Stark Neurosciences Research Institute, Indiana University, 635 Barnhill Dr., MS2049, Indianapolis, IN 46202, USA. ${ }^{14}$ Bakar Computational Health Sciences Institute, University of California San Francisco, San Francisco, CA 94158, USA.

${ }^{15}$ Integrated Cardio Metabolic Centre, Department of Medicine, Karolinska Institutet, Novum, 14157 Huddinge, Sweden.

Published online: 05 November 2019

Reference

1. Glicksberg BS, et al. BMC Med Genet. 2019;12(Suppl 6):108 https://doi.org/1 0.1186/s12920-019-0542-3.

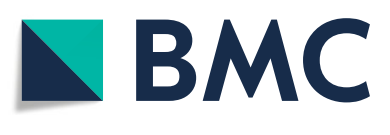

๑ The Author(s). 2019 Open Access This article is distributed under the terms of the Creative Commons Attribution 4.0 International License (http://creativecommons.org/licenses/by/4.0/), which permits unrestricted use, distribution, and reproduction in any medium, provided you give appropriate credit to the original author(s) and the source, provide a link to the Creative Commons license, and indicate if changes were made. The Creative Commons Public Domain Dedication waiver (http://creativecommons.org/publicdomain/zero/1.0/) applies to the data made available in this article, unless otherwise stated. 